\title{
How to approach a research ethics committee
}

\author{
George Masterton \& Prem Shah
}

Abstract This article describes research ethics and the UK research ethics system for psychiatrists who are engaged in research or in supporting trainees with research projects. The emphasis on practical aspects should ensure that submissions to research ethics committees are improved, and consequently the likelihood of a successful outcome is increased.

Applying for ethical approval for a study in the UK can be a daunting task, particularly when confronted with the extensive and detailed submission form. This article is intended to help inexperienced researchers or those supporting them navigate their way successfully through the research ethics system. It is written specifically for those who are planning to conduct a relatively straightforward study or are interested in the processes involved in obtaining ethical consent for research studies in the UK. It does not consider in detail studies with more complex features, such as clinical trials. The reader should go to the National Health Service (NHS) Central Office for Research Ethics Committees website for further advice and for issues not covered here (http:/ / www. corec.org.uk). As in many official areas, abbreviations are ubiquitous. Although we use them sparingly here the most common are listed in Box 1.

Before dealing with the practicalities, we will describe the UK's research ethics system and how it evolved.

\section{Background \\ Establishment}

Research ethics committees were established in response to the Recommendations Guiding Physicians in Biomedical Research Involving Human Subjects that were adopted at the 18th World Medical Assembly held in Helsinki in 1964 (thereafter known as the Declaration of Helsinki; World Medical Association, 2000). The first such committees in the UK were formed in 1967, following a recommendation by the Royal College of Physicians of London that

\begin{tabular}{|c|c|}
\hline COREC & $\begin{array}{l}\text { Central Office for Research Ethics } \\
\text { Committees }\end{array}$ \\
\hline CTIMP & $\begin{array}{l}\text { Clinical trial of investigational } \\
\text { medical products }\end{array}$ \\
\hline MREC & $\begin{array}{l}\text { Multicentre research ethics com- } \\
\text { mittee }\end{array}$ \\
\hline PIS & Participant information sheet \\
\hline REC & Research ethics committee \\
\hline
\end{tabular}

clinical research investigations should be subject to ethical review. Arrangements remained piecemeal and informal until 1975, when the Department of Health introduced a national system which was managed at health authority level and based on the original Royal College of Physicians (1990) recommendations. This was superseded by the 'Red Book' in 1991 (Department of Health, 1991). Since then, the pace of reform and bureaucratisation has accelerated, with research ethics committees being subject to increasing regulation and revision, most recently as a result of the Medicines for Human Use (Clinical Trials) Regulations 2004 (more commonly known as the Clinical Trials Regulations). The entire UK ethics system is now managed and coordinated by the Central Office for Research Ethics Committees, which has established standard operating procedures (all 182 pages of them!) that govern the activities of ethics committees (Central Office for Research Ethics Committees, 2004).

George Masterton is a consultant liaison psychiatrist at the Edinburgh Royal Infirmary (Department of Psychological Medicine, 51 Little France Crescent, Edinburgh EH16 4SA, UK. Email: george.masterton@luht.scot.nhs.uk) and Vice-Chair of the Scottish MREC-A. Prem Shah is a consultant psychiatrist in general adult psychiatry at the Royal Edinburgh Hospital and member of the Scottish MREC-B. 


\section{Criticisms}

The research ethics system has been subjected to repeated criticism. Richard Smith, editor of the $B M J$, chose this topic for his farewell editorial (Smith, 2004). Among the main complaints have been:

- the requirement for duplicate submissions

- submission forms that were long, complex, frequently changed and seemed full of irrelevant enquiries

- excessive delays before a decision was forthcoming

- inconsistent opinions among committees

- interference in the study design.

The difficulties researchers have faced have been well described (Wald, 2004). That the UK may have developed a specific bureaucratic problem was illustrated in a study comparing ethical requirements for a questionnaire-only design submitted in 11 European countries: the UK stood out in terms of the demands placed on the researcher (Hearnshaw, 2004).

Considerable efforts have been made to address researchers' legitimate criticisms. For instance, multicentre research ethics committees were established in 1997 to end the need for duplicate submissions to every ethics committee where a patient might be recruited; the submission form has been electronically refined, with optional sections, better guidance and help; model information sheets and consent forms for participants have been prepared. More recently, an advisory group set up by Lord Warner put forward further proposals that will streamline the process, the core concept being a focus on a more professional system in which the ethics committee is embedded (Department of Health, 2005).

\section{What is a research ethics committee?}

A research ethics committee may be established anywhere in the UK under the governance arrangements for such NHS committees. Currently there are two main types of research ethics committee:

- 'recognised RECs', which are recognised by the UK Ethics Committee Authority under the Medicines for Human Use (Clinical Trials) Regulations 2004 for the purpose of reviewing CTIMPs

- 'authorised RECs', which are authorised by UK health departments or local appointing authorities to undertake the ethical review of health-related research within the NHS either for a particular area or for the entire UK.

\section{Membership}

An ethics committee usually consists of 12-18 people. Up to half are medically qualified, with a mixture of academics and clinicians from a range of specialties. There are nurse members and, ideally, members from other professional groups such as clinical psychologists, social workers and the professions allied to medicine. Lay members, who should account for one-third of the committee and be drawn from a wide social background, are a vital component: they are the element that differentiates research ethics committees from other expert committees that comment on or monitor research. Multicentre research ethics committees employ a professional statistician. All research ethics committees are serviced by an administrative team, who will probably be the researcher's first point of contact.

\section{Procedures and decisions}

The committees meet monthly and as many as 10 new submissions might be considered at a single meeting. Two members, one expert and one lay, act as lead reviewers by providing a comprehensive evaluation. The researcher is invited to attend the meeting, not to give a presentation but to answer enquiries. Attendance often speeds up approval as changes can be agreed and uncertainties resolved immediately. The committee will offer an opinion rather than a decision, with four options currently available to them - favourable, unfavourable, provisional with the outcome depending on further information, and no opinion pending consultation with a referee. The whole procedure from submission to conclusion is strictly time-tabled to take no more than 60 days.

\section{Appeals}

Researchers who receive an unfavourable opinion have the options of re-submitting after addressing the reasons their study was deemed unethical, or appealing, in which case their submission will be reviewed by another committee.

\section{Further contact with the committee}

Following approval the researcher will probably have further contact with the committee. Amendments to an approved design are classified as substantial or minor: substantial amendments require further ethical review and approval, usually by an amendments subcommittee, whereas minor amendments are simply notified to the original 
committee. Distinguishing between substantial and minor amendments is not always straightforward: if in doubt the researcher should consult the committee's administrator, who can refer to the standard operating procedures. Finally, the research ethics committee has a role in monitoring ongoing research. Researchers have a duty to inform the committee of any serious or unexpected adverse reactions and to provide a progress report annually or on completion.

\section{The ethics form}

There is now a single ethics form for the UK, regardless of whether the study involves one or more centres. Although paper copies of the form are available, ethics committees much prefer completed forms to be submitted electronically. Indeed, we advise researchers to complete the form online (http:/ / www.corec.org.uk/applicants/apply/apply.htm), thereby taking advantage of the system's information and help, and saving resources.

The form covers the full range and complexity of ethical issues that may arise in a study. At first it may appear overwhelming, but not all questions will be applicable to all studies, and for simple study designs the system now enables a submission to be completed quickly by bypassing irrelevant sections. For administrative purposes the form is divided into two parts.

\section{Part A}

The first part contains general information necessary for all applications, and it is this part that is of most relevance in our article.

\section{Part B}

Part B asks in detail about important topics that do not apply to all studies, for example medicinal products, ionising radiation and biological materials. Confirmation of the research sites involved and declarations by the chief investigator, sponsor and others as necessary (such as the educational supervisor) also need to be provided here.

\section{Completing the ethics form: frequently asked questions}

\section{What is the first step?}

Before embarking on a research project - that is, before designing the study, never mind submitting it for ethical review - the novice researcher should be

\section{Box 2 Online resources}

British Psychological Society http://www.bps.org.uk (follow links: The Society > Ethics, Rules, Charter, Code of Conduct $>$ Professional Practice Guidelines)

Central Office for Research Ethics Committees http:/ / www.corec.org.uk

Medical Research Council http://www.mrc.ac.uk/PolicyGuidance/ EthicsAndGovernance/index.htm

Royal College of Physicians of London http:/ / www.rcplondon.ac.uk

Royal College of Psychiatrists

Ethics Committee: http://www.rcpsych.ac. uk/college.aspx (follow links: Other resources $>$ Ethics)

World Medical Association

Ethics Unit: http://www.wma.net/e/ethics unit/organizations.htm

familiar with the principles of research ethics (Box 2 gives website addresses for key online resources).

The starting point should be the Declaration of Helsinki (World Medical Association, 2000), which remains the cornerstone of ethical practice in medical research. The Declaration has evolved over 40 years but its format and principles have been maintained (along with its brevity).

Many medical Royal Colleges have published guidance for their members, with the Royal College of Physicians of London producing the first, which is still among the most helpful (Royal College of Physicians, 1996). The Royal College of Psychiatrists published its first guidance in 1989 - in a short and practical but rather idiosyncratic article in the Psychiatric Bulletin (Royal College of Psychiatrists, 1990). This covered some of the specific problems likely to be encountered in mental health research, for example detained patients and 'incompetent' adults. It was superseded by a Council Report prepared by a distinguished committee and authoritatively covering all aspects of the topic both for researchers and research ethics committees (Royal College of Psychiatrists, 2001). Reading and assimilating this report is a must for all aspiring psychiatric researchers and those supporting them, and a copy should be available for reference.

The British Psychological Society recently revised its original 1978 guidelines and embedded these in its Code of Conduct (British Psychological Society, 2006), thereby positioning this topic more centrally than other professional groups have done. 
The Medical Research Council has a long record of providing authoritative opinion on ethical aspects of research. It published helpful guidelines on research on the mentally incapacitated in 1991, which were updated in 1993 (Working Party on Research on the Mentally Incapacitated, 1993). Its website (see Box 2 for url) maintains the MRC Ethics Series of articles on many aspects of its published research.

\section{Is ethical approval needed?}

It is important to differentiate between research and audit or service evaluation. All medical research taking place in the UK has to obtain a favourable opinion from a research ethics committee before proceeding, whereas audit and service evaluation do not. Fundamentally, research is concerned with the right thing to do and audit with ensuring that it is being done right, but this distinction is often unclear (Wilson et al, 1999). The Central Office for Research Ethics Committees has devised a table to assist researchers in distinguishing research from other activities (Table 1). It must be emphasised that these definitions are not watertight, and there are plenty of grey areas. If in doubt, individuals planning a research study should contact their research ethics committee administrator. Generally, if uncertainty persists the proposal should be processed as a research study, as this provides safeguards for all concerned (and ultimately it may make the difference between a journal accepting or rejecting the paper).

Ethical approval is required for any research study in the UK if it involves:

- patients or users of the NHS

- relatives or carers of patients or users of the NHS

- access to data, organs or other bodily material of past or present NHS patients
- foetal material or in vitro fertilisation involving NHS patients

- the recently dead on NHS premises

- the use of, or potential access to, NHS premises or facilities

- NHS staff recruited as research participants by virtue of their professional role.

\section{How should the application process be approached?}

\section{Leave sufficient time}

Submitting an ethical approval form can be timeconsuming and should never be left to the last minute: this is the most common error made by inexperienced researchers. Time is needed not only to complete the form - there is other documentation to submit (such as participant and/or relative/ general practitioner information sheets; consent forms; advertisements; the protocol; scientific critiques/reviews; permissions). This stage should be approached as a serious endeavour that may well improve the study, rather than as an unnecessary hurdle.

Get advice on how to submit, and understand the local arrangements

The national structure is now well organised through the Central Office for Research Ethics Committees, which provides helpful online guidance about the requirements for submission (http://www.corec. org.uk/applicants/apply/apply.htm\#guidance). However, local arrangements still vary considerably, and applicants are strongly advised to explore this at an early stage. A good source of information for trainees will be their colleagues who have recently obtained ethical approval or are further along the

\section{Table 1 Differences between research, clinical audit and service evaluation ${ }^{1}$}

Research
Designed and conducted to generate
new knowledge

Quantitative research: hypothesis based

Qualitative research: explores themes following established methodology

Addresses clearly defined questions, aims and objectives

May involve a new treatment

May involve additional therapies, samples or investigations

\section{Clinical audit}

Designed and conducted to provide new knowledge to provide best care

Designed to answer the question:

'Does this service reach a

predetermined standard?'

Measures against a standard

Does not involve a new treatment

Involves no more than administration of questionnaire or analysis of records

\section{Service evaluation}

Designed and conducted to define current care

Designed to answer the question: 'What standard does this service achieve?'

Measures current service without reference to a standard

Does not involve a new treatment

Involves no more than administration of simple interview, questionnaire or analysis of records

1. Modified from Central Office for Research Ethics Committees (2006). The full table can be seen at http://www.corec.org.uk/ applicants/help/docs/Audit_or_Research_table.pdf 
process of an active submission: learning about how the system works locally, seeing a completed ethics form and discussing the practicalities of the process will save time and worry. For questions that fellow researchers cannot answer the applicant could approach either the ethics committee administrator, or their NHS trust or health authority research and development department/office, or a senior colleague who is a seasoned researcher and/or who serves on an ethics committee.

\section{Seek peer review for the study}

Externally funded projects will have undergone independent peer review as a matter of course during the funding application process (although the scientific quality of this varies greatly). However, for projects with no funding attached and/or for small-scale projects, internal review is fine. In these circumstances review could be carried out by the head of department or an appropriate, experienced researcher: many academic departments have established their own peer review group for this purpose. Where no academic peer group is available, a local journal club is another option.

On the form communicate clearly and in straightforward, non-technical terms

Ethical approval will be delayed if the study cannot be understood, or if it is inadequately or confusingly presented. Pasting in chunks of the protocol should be avoided - remember that the applicant is communicating with a different audience for a different purpose.

\section{Establish funding}

Preferably, funding should be secured before submission for ethical approval. However, ethics committees recognise that researchers can face a chicken and egg situation, as some funding bodies require committee approval before they will consider a grant application. Obtaining ethical approval before grant submission may necessitate re-submission to the committee if the funding body requires major design changes. If the application is submitted for funding first, it is important to remember that the subsequent ethics process may delay the project start date. If the project involves no additional funding this should be stated on the form.

\section{Establish the sponsor}

A sponsor is an organisation, represented by a nominated individual, that ensures the appropriate arrangements are in place for the initiation, management and financing of the study. It may be the funding body, although more often it will be

\section{Box 3 Negligent and non-negligent harm}

Negligent harm is the legal liability that arises when a participant is harmed by an act of negligence, for example if the researcher did not abide by procedures required in the study protocol. NHS indemnity arrangements will cover only negligent harm.

Non-negligent (no-fault) harm arises when a participant has been harmed in circumstances where nobody involved in the research has been at fault, for example by a completely unexpected drug reaction. Non-negligent harm is not covered by NHS indemnity, so researchers must arrange their own cover through medical defence unions or private insurance schemes if there is a recognised risk. In practice this is a remote occurrence outwith interventional studies.

the applicants employer, for example their trust or university. Applicants need confirmation from the sponsor that they have agreed to accept this responsibility. The relevant NHS trust or health authority research and development department/ office often adopts this role. Even if another body is sponsor it will be necessary to contact the research and development department to ensure that the study can be hosted if NHS premises or resources are involved.

\section{Confirm arrangements for insurance/indemnity}

Detail the insurance/indemnity arrangements that are in place for negligent and non-negligent harm (Box 3). These arrangements will depend on the type of research: with drug trials, for example, Association of the British Pharmaceutical Industry (ABPI) guidelines are usually operative.

\section{To what areas does the committee pay particular attention?}

Study design

There is an active debate about whether research ethics committees have any right to seek changes to, or even comment on, the design of a proposed study, a topic that is aired in the Warner Report (Department of Health, 2005). The problem is that science and ethics are not mutually exclusive, so that design considerations such as the exclusion of minority ethnic groups from research, the inclusion of a placebo control group when standard therapy is available and the availability after the trial ends 
of a treatment found to be effective have ethical dimensions. Usually a design that has been properly peer reviewed will pass unscathed, whereas a project that has not, particularly if submitted by an inexperienced researcher without adequate supervision, will probably run into difficulties and may be rejected on scientific grounds alone. That said, research ethics committees may still take a different view from expert scientific bodies about key design issues because the committee's first duty is to the individual participant rather than the scientific merit or the greater good.

\section{Recruitment issues}

It is important to give full details of how participants will be identified (i.e. without breaching the requirements of the Data Protection Act 1998), approached and recruited. The committee needs to establish how this will work in practice, so simply writing 'from the clinic' will not suffice. If the research is derived from data stored on a database, the applicant will need the appropriate permissions to access it. Copies of any advertisements for participants, with a justification for this method of recruitment, should be included with the application.

\section{Data analysis}

The applicant may need to provide confirmation that they have received valid advice regarding their statistical methodology, particularly with regard to statistical power, sample size and analysis strategy. Remember, there is a professional statistician on many research ethics committees! If a statistical expert has not been consulted, this should be justified.

\section{Participant information sheets}

Unsurprisingly, ethics committees pay very close attention to the information that will be given to the participant or their representative, as this is the basis for obtaining informed consent. It is particularly important that this information is imparted in lay terms. A 'question and answer' format is often best. We recommend that novice researchers ask one or more lay people to read through their participant information sheet to ensure that it is easy to understand before it is submitted to the committee. We also strongly advise using COREC's helpful guidance for producing a participant information sheet (http://www.corec.org.uk/applicants/help/ docs/Guidance_on_Information_Sheets_and_ Consent_Forms.doc).

\section{Consent forms}

The committee will also concentrate on consent - considering both the process of consent and the wording of the form. Problematic issues include when participants are to be given very little time to consider the participant information sheet, when proxy consent is necessary, when consent is to be provided orally or when no consent at all is to be sought. Consent may be required for specific aspects of the study (such as a video recording, access to medical notes, sending data outside the European Union). We recommend the template consent form at the COREC link given at the end of the previous paragraph.

\section{Care and protection of participants}

The committee needs to know what research-related interventions are to be carried out and, crucially, to see that the potential risks of the study have been identified and quantified. If normal treatment is being withheld or altered this must be justified fully. Flow diagrams or timetables are good ways of explaining complicated or potentially arduous procedures. Because of the problem of overstudying patients, particularly if they have unusual or topical disorders, the proposed study should be placed in the context of other studies that the participant may be in shortly before, during or just after the proposed study.

\section{Coercion of participants}

One of the fundamental duties of the committee is to ensure that individuals are free to participate or not as they choose, without undue influence. The abuse of people by doctors and scientists to further their research is not confined to the archetypal evil perpetrated by the Nazis. The infamous Tuskegee syphilis study, for example, demonstrated that even in democratic societies such as the USA unconscionable (if unintended) abuse could occur (Bhopal, 1997; Centers for Disease Control and Prevention, 2005). The Declaration of Helsinki makes a key ethical distinction between research and research that is combined with clinical care, to take account not only of undeniable abuse but also of the actions of the well intended. Researchers need to think carefully about whether their role as a patient's physician may compromise that patient's freedom of choice when the roles switch to researcher and participant. Ethics committees will also pay close attention to overt inducements to participants, such as financial rewards or unjustified promises of improvement. They are concerned too about the integrity of vulnerable participants such as people with mental disorders and prisoners. The provision of an independent person whom the participant or their family can approach to seek impartial advice is sometimes encouraged. 
Community, confidentiality and data protection issues

The committee will consider whether and how participants and their community (if appropriate) have been consulted in the study design, and whether or how findings, especially individual results, are fed back. Participants' data must be stored securely and their anonymity maintained. Incidentally, for research confined to examination of case records or databases, i.e. when there is no direct contact with patients, submission to an ethics committee will still be necessary if the project does not satisfy the criteria for audit.

Whether medical information held on national databases can be released to researchers is determined by the advisory group for medical research of the Office for National Statistics in England and Wales, and the Privacy Committee in Scotland. At local level, the NHS board or trust's Caldicott guardian and its data protection officer ensure that personal health data are released to researchers in accordance with the Data Protection Act 1998. There have been serious concerns raised recently about how these authorities are impeding epidemiological research in particular (Iversen et al, 2006; Walley, 2006).

\section{What should be done if the study may involve people with impaired capacity to consent?}

There are now legal requirements in the UK when undertaking research on adults who are unable to provide informed consent. In Scotland, a national research committee was established in 2002 under Section 51 of the Adults with Incapacity Act (Scotland) 2000 to consider all medical research to be conducted in Scotland that included any adults lacking capacity. The Mental Capacity Act 2005, which regulates treatment and research on adults without capacity in England and Wales, has not followed this degree of specialisation, although it does share basic principles that extend to consideration of research (Box 4). These requirements do not apply to studies conducted under the Clinical Trials Regulations - thus paradoxically affording less protection to adults with incapacity entered into drug trials, where the need for enhanced protection of vulnerable participants is arguably greatest.

\section{Conclusions}

Seeking ethical approval for a project should not be regarded as a distraction, an obstacle or an irritant. Research ethics committees were established primarily to protect patients from bad research, but
Box 4 Summary of conditions governing research on adults who lack capacity in the UK

- The research must be into the causes, diagnosis, complications, treatment, care and/or outcome of the disease that affects the participant or their family

- It is not possible to reach a meaningful conclusion regarding the outcome of the study without including adults who lack capacity

- The risks and non-hazardous harms must be negligible if there is no prospect of benefit for the individual participant, and clearly outweighed by the potential for good if there is

- The participant must not object

- Consent must be obtained from a person who is authorised to provide proxy consent under the legislation

they have a secondary duty to foster good research. Research is enhanced by independent consideration of its ethical dimension, in particular through focusing on the participant's experience.

\section{Declaration of interest}

None.

\section{References}

Bhopal, R. (1997) Is research into ethnicity and health racist, unsound, or important science? BMJ, 314, 1751-1756.

British Psychological Society (2006) Ethical Principles for Conducting Research with Human Participants. British Psychological Society.

Centers for Disease Control and Prevention (2005) 'The Tuskegee Timeline'. CDC. http://www.cdc.gov/nchstp/od/tuskegee/ time.htm

Central Office for Research Ethics Committees (2004) Standard Operating Procedures for Research Ethics Committees in the United Kingdom (Version 2.0). COREC.

Central Office for Research Ethics Committees (2006) 'Differentiating Audit, Service Evaluation and Research'. COREC. http://www.corec.org.uk/applicants/help/docs/ Audit_or_Research_table.pdf

Department of Health (1991) Guidelines to Local Research Ethics Committees. TSO (The Stationery Office).

Department of Health (2005) Report of the Ad Hoc Advisory Group on the Operation of NHS Research Ethics Committees (Report 268110). Department of Health.

Hearnshaw, H. (2004) Comparison of requirements of research ethics committees in eleven European countries for a noninvasive, interventional study. BMJ, 328, 140-141.

Iversen, A., Liddell, K., Fear, N., et al (2006) Consent, confidentiality and the Data Protection Act. BMJ, 332, 165-169.

Royal College of Physicians (1990) Guidelines on the Practice of Ethics Committees in Medical Research Involving Human Subjects (2nd edn). Royal College of Physicians of London. 
Royal College of Physicians of London (1996) Guidelines on the Practice of Ethics Committees in Medical Research Involving Human Subjects (3rd edn). Royal College of Physicians of London.

Royal College of Psychiatrists (1990) Guidelines for Research Ethics Committees on psychiatric research involving human subjects. Psychiatric Bulletin, 14, 48-61.

Royal College of Psychiatrists (2001) Guidelines for Researchers and for Research Ethics Committees on Psychiatric Research Involving Human Participants (Council Report CR82). Royal College of Psychiatrists.

Smith, R. (2004) My last choice. BMJ, 329, 0-g. doi: 10.1136/ bmi.329.7460.0-g

Wald, D. (2004) Bureaucracy of ethics applications. BMJ, 329, 282-285.

Walley, T. (2006) Using personal health information in medical research. $B M J, 332,130-131$

Wilson, A., Grimshaw, G., Baker, R., et al (1999) Differentiating between audit and research: postal survey of health authorities' views. BMJ, 319, 1235.

Working Party on Research on the Mentally Incapacitated (1993) Ethical Conduct of Research on the Mentally Incapacitated. MRC. http://www.mrc.ac.uk/Utilities/Documentrecord/index. htm?d=MRC002409

World Medical Association (2000) Ethical Principles for Medical Research Involving Human Subjects. WAM. http:/ / www.wma. net/e/policy/b3.htm

\section{MCQs}

1 The following indicate research rather than audit:

a an additional blood test

b questionnaires

c seeks best care as the primary outcome

d does not involve a new treatment

e assessing service user satisfaction.

2 Incapacity legislation and research in England and Wales:

a applies only to mental disorders

b differs in Scotland

c applies to clinical trials

d excludes detained patients from research

e applies to children.
3 Research ethics committees:

a should review all NHS research

b must have $50 \%$ lay membership

c must process a submission within 100 days

$\mathrm{d}$ have no role in monitoring research

e are all empowered to approve any research project.

4 The Declaration of Helsinki:

a concerns global warming

$\mathrm{b}$ is confined to therapeutic research

c focuses on mental health research

d focuses on research combined with clinical care

e is legally binding in the UK.

5 An authorised research ethics committee can consider research that involves:

a gene therapy

$\mathrm{b}$ adults lacking capacity in Scotland

c healthy volunteers in a phase 1 clinical trial

$\mathrm{d}$ patients detained under mental health legislation

e a phase 3 clinical trial of a medicinal product. 